\title{
The Formation of Carbon in Combustion and how to Quantify the Impact on Human Health
}

\author{
K. Siegmann and H.C. Siegmann \\ Swiss Federal Institute of Technology, Zürich
}

\begin{abstract}
We summarize our current research on combustion aerosols. First, sampling devices for the analyses of flame gases are described. The flame gas samples are investigated by mass spectroscopy and by standard aerosol techniques. Time-of-flight mass spectroscopy is well suited to study formation and growth of soot precursor molecules. Fullerenes can also be seen in some mass spectra of flame gases. Presumably, the fullerenes are evaporated from small soot particles in the mass spectrometer by the ionizing laser. Size spectra of soot particles from the flame are presented. The flame is optionally seeded with palladium aerosol to demonstrate that the particle size distribution is not altered during the sampling procedure. It is found that soot particles are already present low in the flame where large molecules are absent.

Photoemission is applied to study surface properties of soot particles from the flame. It is shown that the surface of the particles is covered with polycyclic aromatic hydrocarbons (PAH). The PAH can be removed by heating and the properties of the carbon core are revealed. One can thereby distinguish a soot growth from a soot burnout region in the flame. Time-resolved desorption experiments of perylene (a PAH) from model aerosol particles are presented. It is shown that they follow a first order rate law. The photoelectric $\mathrm{PAH}$ sensor is introduced as a personal air quality monitor. The danger from inhaling combustion aerosol can be expressed in units of standard cigarettes.
\end{abstract}

The carbon atom has the unique capability of forming clusters at temperatures above $1000^{\circ} \mathrm{C}$. Such temperatures occur in the combustion of organic materials and fuels used almost exclusively in our present civilisation for power generation. The carbon clusters are very difficult to get rid of once they have formed. As the exhaust gas carrying the carbon clusters cools down, various other chemicals adsorb onto the carbon clusters arising from wear and tear in the engine, or synthesized in the process of combustion, or simply from unburnt fuel. In this way, the carbon clusters become the skeleton of the soot particles found suspended in great quantities in the air in which we live. The soot particles are of respiratory size, that is they are deposited in the human respiratory tract where they cause life-threatening diseases. It has been shown in the famous Harvard six cities study [1], and subsequent similar work in 15 European cities, that mortality from cardiopulmonary disease increases by $1 \%$ when loading of the air with respiratory particles increases by $10 \mu \mathrm{g} / \mathrm{m}^{3}$. Even in allegedly clean cities, such as the city of Zurich, 3-4 times higher concentrations of respiratory particles are typically generated by automotive traffic alone. Moreover, it is very well known that there are also long-term health risks associated with the inhalation of respiratory

particles; lung cancer is the most dreadful of them. There is a delay of as much as 2030 years between the beginning of the exposure to the soot particles and the outbreak of the disease, and this delay very often leads younger people to believe that they are immune, despite the clearcut epidemiological evidence. However, very recently all doubts have been removed, as a direct etiological link between benzo(a)pyrene ( $\mathrm{B}(\mathrm{a}) \mathrm{P})$ and human lung cancer has been established using the methods of molecular biology [2]. $\mathrm{B}(\mathrm{a}) \mathrm{P}$ belongs to the class of polycyclic aromatic hydrocarbons (PAH). These chemicals are at the centre of our work. We will show how $\mathrm{PAH}$ are synthesized in combustion concomitantly with the carbon clusters. We will also show that the heavier PAH, including the carcinogenic species such as
$\mathrm{B}(\mathrm{a}) \mathrm{P}$, are adsorbed on particles at ambient temperature. Lastly, we will demonstrate that respiratory particles having adsorbed PAH on their surface are detected readily by the fact that they can be charged electrically with great efficiency through photoemission of electrons. Based on these findings, we determine the individual human exposure to particles from combustion and relate this exposure to that received when smoking a cigarette. It turns out that for many people the dose of $\mathrm{B}(\mathrm{a}) \mathrm{P}$ received from traffic fumes is equivalent to smoking a few cigarettes every day.

We are not the only people worried about the health impact of particles from combustion. Mario Molina, winner of the chemistry Nobel prize in 1995 for his work on atmospheric ozone depletion, gave part of his prize money to a foundation supporting research on air pollution in Latin America [3]. At the first world congress on air pollution in San José in Costa Rica in November 1996, it was estimated that 460000 people die worldwide every year from respiratory particles produced by automotive traffic. This figure shows that the danger of smoke from traffic now approaches the danger from cigarette smoke. The latter is estimated to kill more than 3 million people a year.

Fine particles from combustion contain thousands of different chemicals. Complete chemical characterization is impossible because of this complexity, the very tiny amount of material, and the

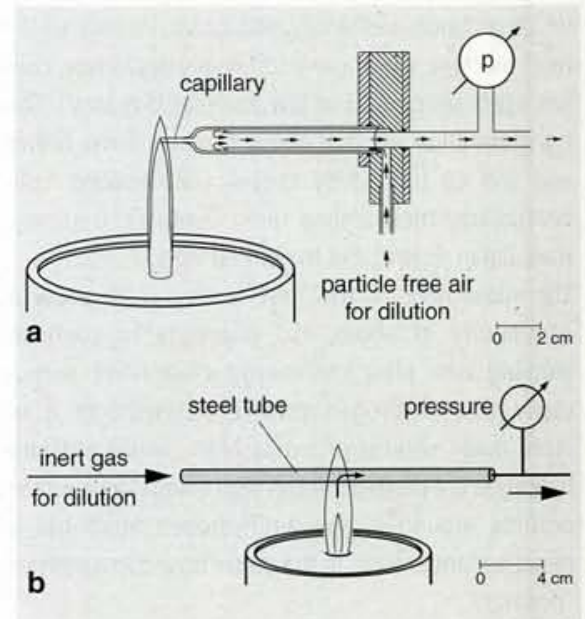

Fig. 1. Sample extraction systems.

a) The ceramic capillary is glued to a double-walled quartz tube. The dilution gas enters through the annular space between the tubes.

b) The flame gases enter through the hole in the bottom of the tube in which the dilution gas flows. 
chemicals that are subject to change in the atmosphere. However, common to all particles from incomplete combustion is the carbon skeleton. Cluster science has developed the tools to characterize clusters and to understand their formation on the atomic and molecular level. Therefore, research on the formation of carbon in combustion is at the forefront of the research in solid state physics, and also is the most obvious application of cluster science to which K.H. Bennemann has significantly contributed.

\section{Sampling from inside the com- bustion zone}

The present work differs from the work of others in that we take samples of gas containing molecules and particles directly from the combustion zone. The small sampled volumes are immediately and highly diluted with cold inert gas such as $\mathrm{Ar}$ or $\mathrm{N}_{2}$ and subsequently fed into a time-of-flight (TOF) mass spectrometer. Simultaneously, aerosol sizing equipment, including an interaction tube with the light from pulsed lasers, is used to determine the size distribution and surface properties of the particles. For the most important case of the $\mathrm{PAH}$, the enthalpy of adsorption and the time needed to reach the desorption/ adsorption equilibrium at the particle surface is also measured. As a combustion model, we have chosen a laminar methane diffusion flame. However, the technique may be applied to almost any combustion, be it wood fire, diesel engine, cigarette, or whatever else one likes to imagine. Yet the model we present here has some advantages for developing the basic understanding of carbon formation in combustion. First, methane $\left(\mathrm{CH}_{4}\right)$ is the simplest organic fuel. It produces comparatively few soot particles which are all burnt up before the combustion gases cool down. The small particle concentration means that interaction between particles, such as agglomeration, is minimal. Second, a time scale is established by the laminar flow. The distance from the orifice of the burner can be translated into time spent in the combustion zone. As the oxygen is supplied by diffusion from the outside, the symmetry axis in the middle of the flame has the lowest oxygen partial pressure. The results discussed below are valid for the symmetry axis of the cylindrical flame. To avoid flickering of the flame, it is surrounded by a laminar flow of air.

In Fig. 1 we show a comparison of the two sample extraction systems used. In
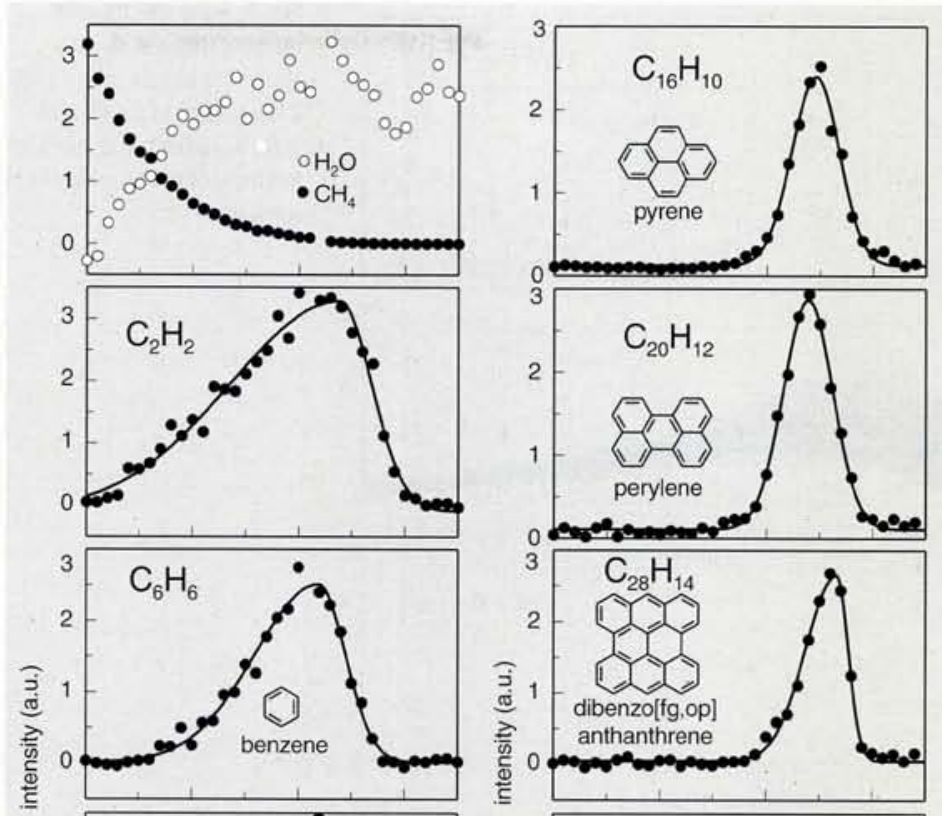

Fig. 2: Height profiles of selected molecules found in the flame. $\mathrm{CH}_{4}, \mathrm{H}_{2} \mathrm{O}$, and $\mathrm{C}_{2} \mathrm{H}_{2}$ were ionized by electron impact, the other molecules which are polycyclic aromatic hydrocarbons (PAH) by ionization with an excimer laser of $5 \mathrm{eV}$ photon energy. The intensities depend on the ionization efficiency which is different for each molecule.
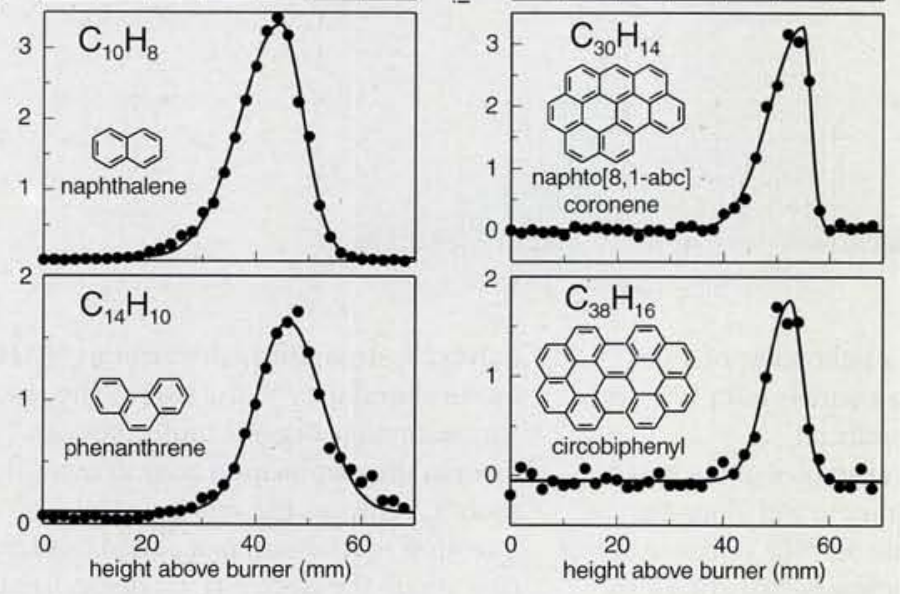

system a) a capillary of ceramic material extends into the flame. Flame gases are extracted by maintaining reduced pressure in the quartz tube to which the capillary is attached. The flame gases expand into the quartz tube where they are diluted with nitrogen or argon by factors ranging from 10-1000, depending on the experiment. The capillary does not visibly disturb the flame. The position of the capillary orifice in the flame is adjusted by moving the flame with a computer-driven mechanical motion. It is thus possible to take samples from any point in the flame. We have reason to believe that the height resolution of the sampling is less than $0.1 \mathrm{~mm}$ while we do not know the exact radial extension of the sampled volume. In system b) a tube in which the inert dilution gas flows is placed across the flame. Depending on the pressure difference between flame and the dilution gas stream, flame gases enter into the tube through a pinhole at the bottom. This device disturbs the flame, but essentially only after the sample has been taken. Its advantage compared to a) is that it is simpler to construct and it samples with superior radial resolution. We have not observed any systematic differences between samples taken using system a) or b) $[4,5]$.

Time-of-flight (TOF) mass spectroscopy with pulsed laser ionization

The diluted flame gases pass by a pulsable valve which opens for a few microseconds, allowing the gases to expand into the vacuum chamber of a commercial Bruker-Franzen TOF mass spectrometer. The skimmer defines a beam in which the temperature is low due to the supersonic expansion. The light molecules such as $\mathrm{H}_{2} \mathrm{O}, \mathrm{CH}_{4}$, or $\mathrm{C}_{2} \mathrm{H}_{2}$ are ionized by electron impact for TOF mass spectroscopy. With the PAH, ionization by photoemission of electrons is used to avoid fragmentation. 


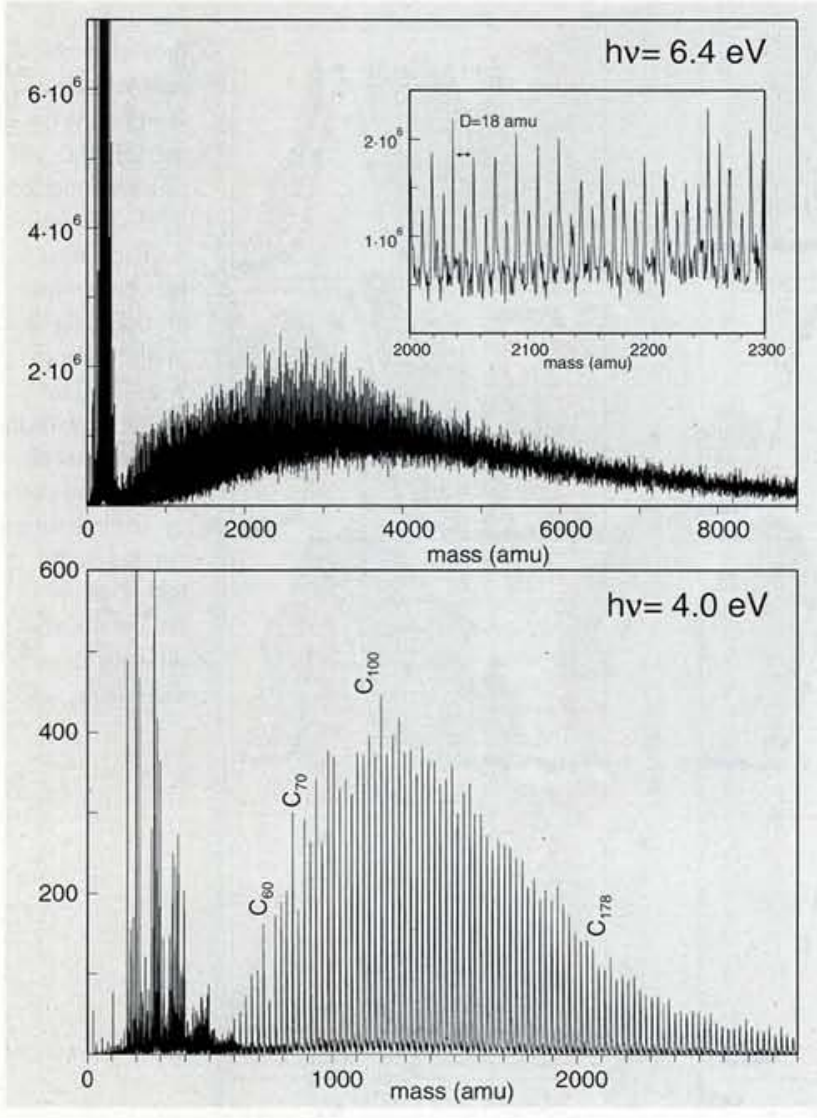

To this purpose, a light pulse of $20 \mathrm{~ns}$ duration from an excimer laser is fired across the supersonic jet.

Fig. 2 shows examples of the height profiles of molecules found along the centre of the flame. For the purpose of this work, it is not necessary to distinguish between various isomers. It should be noted that the visible height of the flame is $70 \mathrm{~mm}$; one observes that the concentration of all the hydrocarbons is zero at that height. The concentration of two different species cannot be compared as the detection efficiency is different for each molecule. The concentration of the fuel $\mathrm{CH}_{4}$ decreases sharply with height above the burner orifice; it is already at the $10 \%$ level at $\mathrm{h}=20 \mathrm{~mm}$. The concentration of the main combustion product $\mathrm{H}_{2} \mathrm{O}$ increases at the corresponding rate. Hence the main energy-producing process is already terminated after about $20 \mathrm{~mm}$. The remaining $50 \mathrm{~mm}$ of the flame are apparently needed to form and destroy a rich variety of $\mathrm{PAH}$ and carbon clusters. Acetylene $(\mathrm{HC} \equiv \mathrm{CH})$ with a triple carbon bond is present almost everywhere in the flame. Its concentration reaches a flat maximum at around $50 \mathrm{~mm}$ height. It is very plausible, as many authors have proposed, that acetylene is the main building block of the large and flat
Fig. 3: Mass spectra with samples taken at $55 \mathrm{~mm}$ above the burner orifice with a laser beam of $6.4 \mathrm{eV}$ and $4.0 \mathrm{eV}$ photons. The average intensity of the laser was kept constant.

polycyclic aromatic hydrocarbons $(\mathrm{PAH})$ found abundantly in the flame. The reason why such huge organic molecules can form in the combustion zone at more than $1000^{\circ} \mathrm{C}$ relies on the very principles of quantum mechanics: in a closed benzene ring where the electrons are delocalized, no boundary conditions exist to exclude the constant as a possible wave function. Hence two electrons can be accommodated with zero kinetic energy. This is not possible in linear molecules which are therefore less robust at high temperatures. It is interesting to note that $\mathrm{PAH}$ are also thought to be synthesized when an oxygen-deficient red giant expands in the cosmos [6]. An important conclusion from the height profiles shown in Fig. 2 is that the larger PAH are successively built from smaller units. While benzene occurs over a sizeable range of heights, the concentration profiles sharpen and their maxima shift to greater heights as one moves to the larger PAH. Yet we have observed that some PAH occur lower in the flame than expected from their size. This happens for those species that are built from two small ones under dehydrogenation [7]. As an example, we mention perylene which is obtained by adding two naphthalenes and releasing $\mathrm{H}_{2}$. We note that we have detected the largest PAH ever found in combustion, containing over $50 \mathrm{C}$ atoms in 20 coupled benzene rings. We have also found curved PAH containing carbon rings with five corners which create dishes [7].

Extending the range of atomic mass units to $10^{4}$ reveals new phenomena. Fig. 3 shows that $\mathrm{PAH}$ and their fragments come to an end at around $600 \mathrm{amu}$. New entities appear after a clearcut minimum. The observed mass spectra depend on the photon energy hv. They are, apart from a superimposed beat structure, featureless if $h v=6.4 \mathrm{eV}$. But with $\mathrm{h} v=4 \mathrm{eV}$, the spectra show a rich variety of fullerenes and their fragments. This occurs for the identical average intensity of the excimer laser beam. The spectacular phenomenon is explained if one assumes that the masses detected in the 1000-10000 amu range belong to carbon clusters. The photoelectric work function $\Phi$ of these clusters is larger than $4 \mathrm{eV}$ but smaller than $6.4 \mathrm{eV}$. Hence for $\mathrm{hv}=6.4 \mathrm{eV}$, the particles are charged by photoelectron emission which is impossible at $h v=4 \mathrm{eV}$. To understand charging at $4 \mathrm{eV}$, one must take into account that the intensity in a light beam from an excimer laser exhibits hot spots. We have shown that thermionic emission of electrons occurs from the particles in the hotspots [8]. With $\mathrm{h} v=4 \mathrm{eV}$, the dominant charging mechanism is by thermionic emission. This mechanism selects the particles exposed to the hot spots for detection. Fig. 3 proves that fullerenes and their fragments evaporate from those hot carbon clusters. Concluding the discussion of the phenomena in the range of the carbon clusters, we mention that a closer analysis of the beat structure obtained with $h v=6.4 \mathrm{eV}$ might lead to deeper insight into the structure of the carbon clusters. The mass ratio of the $\mathrm{C}$ atom to the $\mathrm{H}_{2} \mathrm{O}$ molecule is 2:3. If carbon clusters were added in units of three carbon atoms, two adsorbed water molecules would be identical to adding one carbon unit, and a mass spectrum with peaks every $18 \mathrm{amu}$ would appear, disturbed by the isotope effect as observed.

The question now arises whether the fullerenes can be generated from any kind of carbon particle. Fig. 4 suggests that this is not the case. In this figure, the height profile of the PAH $\mathrm{C}_{13} \mathrm{H}_{10}$ and the height profile of the prototype fullerene $\mathrm{C}_{60}$ are shown. In contrast to the PAH, the fullerenes all show identical height profiles independent of their mass [9]. First, this key observation proves that the fullerenes 


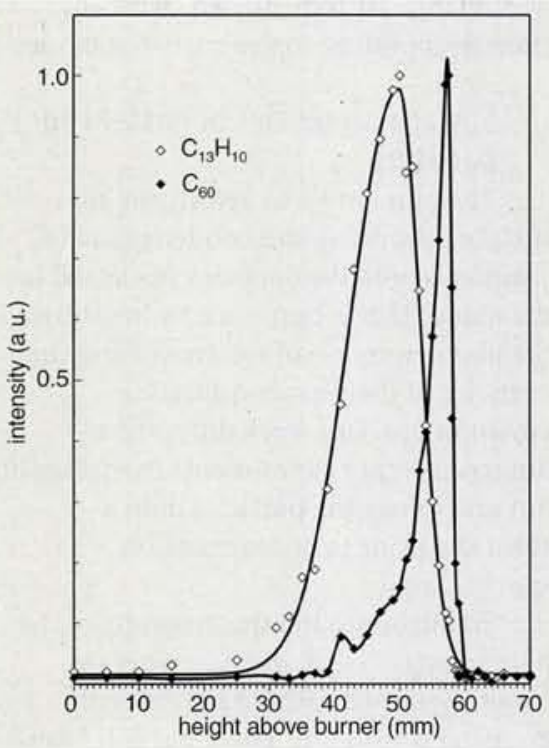

Fig. 4: Height profile of the PAHC $C_{13} \mathrm{H}_{10}$ and of the fullerene $\mathrm{C}_{60}$.

are not built up successively from smaller units as is the case with the PAH. Second, as the particles are still fully developed even at $70 \mathrm{~mm}$ (see below), only the special particles found at or below $60 \mathrm{~mm}$ can emit fullerenes.

The conclusion from this height profile is that the fullerenes exist within the type of carbon cluster present at $60 \mathrm{~mm}$. The fullerenes evaporate upon heating. It is possible that heating with the laser beam supports or completes the formation of the fullerenes. We should point out here that it is likely that the method of TOF spectroscopy after laser ionisation induces changes in the sample. A standard method of manufacture of fullerenes is laser excitation followed by expansion or cooling. We draw attention to the sharp upper boundary for the existence of the fullerenes. The sharpness of this feature proves the excellent height resolution of the sample extraction system.

To connect these findings with the large body of existing literature on soot formation, we shall discuss the standard model of soot formation put forward by a number of authors $[10,11]$. According to this model, the first step is that the hydrocarbon fuel is broken up into small radicals from which acetylene and benzene are synthesized. After that, larger $\mathrm{PAH}$ are successively built from benzene and acetylene, or by polymerization under dehydrogenation. The PAH can be flat or curved depending on the author. It is then supposed that the large $\mathrm{PAH}$ agglomerate by van der Waals forces and/or occasional $\mathrm{sp}^{3}$ bonds to form the first carbon clusters.

Although some observations made here, such as the successive build-up of $\mathrm{PAH}$ and the depletion valley in the mass spectra before the onset of the carbon clusters, seem to favour the standard model of soot formation, we will show below that this model cannot really be the right one as the first particles appear much before the heavy PAH have reached their maximum concentration. To detect the height at which the famous soot inception point is actually located, one needs to employ the far more sensitive aerosol techniques introduced below. The results from these techniques suggest that at least the large PAH are a byproduct rather than the cause of carbon cluster formation.

\section{The size distribution of the par- ticles in the combustion zone}

Figure 5 shows the setup needed to measure the size distribution of gas suspended particles and to determine their interaction with the light emitted by a laser, for example.

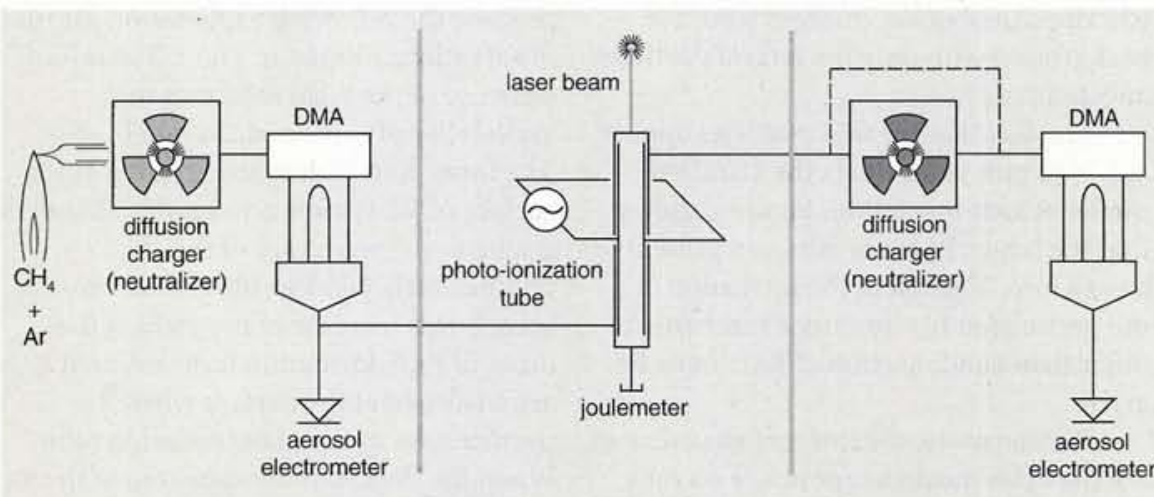

Fig. 5: Experimental setup for the measurement of size distributions and surface properties of gas suspended particles. DMA is the differential mobility analyzer.

The gas stream carrying the particles first enters a diffusion charger. This device contains a radioactive source which produces positive and negative charge carriers in the gas with the particles. If the particles are already charged upon entering, this charge will attract the opposite charge and be neutralized. After some time, the equilibrium charge will be established on the particles. With particles of radius $\mathrm{r}<100 \mathrm{~nm}$, most will remain neutral since the electrostatic energy $\mathrm{e}^{2} /\left(4 \pi \varepsilon_{0} \mathrm{r}\right)$ exceeds the thermal energy $\mathrm{kT}$. About $1 \%$ of the particles carry one elementary charge. Doubly-charged particles are rare.

After the diffusion charger, the gas carrying the particles enters the differential mobility analyzer (DMA). It selects particles with one electrical mobility. To the extent that the assumption of singly-charged particles holds, this also means that particles of one specific mobility diameter $\mathrm{d}=2 \mathrm{r}$ are separated. If the particles are not spherical in shape, the mobility diameter is the diameter of the sphere with the same electrical mobility as the particle. After the DMA, the gas stream then carries monodispersed singly-charged particles. The intensity of this particle current is measured by letting the carrier gas pass through a filter which catches all particles. The electric current flowing from the filter to ground potential is measured with a femto-amperemeter. By varying the voltage on the DMA, particles of different electrical mobility are selected. Plotting the current in the filter against the voltage applied to the DMA, the size distribution of the suspended particles is obtained. All this is by now standardized, commercially available equipment. We shall call the filter with the amperemeter "the aerosol electrometer".

To measure the photoelectric yield of the particles, the gas carrying the sizeselected particles flows through a tube before entering the aerosol electrometer. The sample is exposed to ultraviolet light in the tube. The photoelectrons emitted from the particles are removed by an alternating electric field. This field precipitates the electrons at the electrodes, whereas it leaves the particles that emitted the photoelectrons within the gas stream. The differential current measured behind the tube in the aerosol electrometer with the light switched on and off is proportional to the photoelectric yield. If one wants to observe any changes of particle size due to interaction with the light, a 


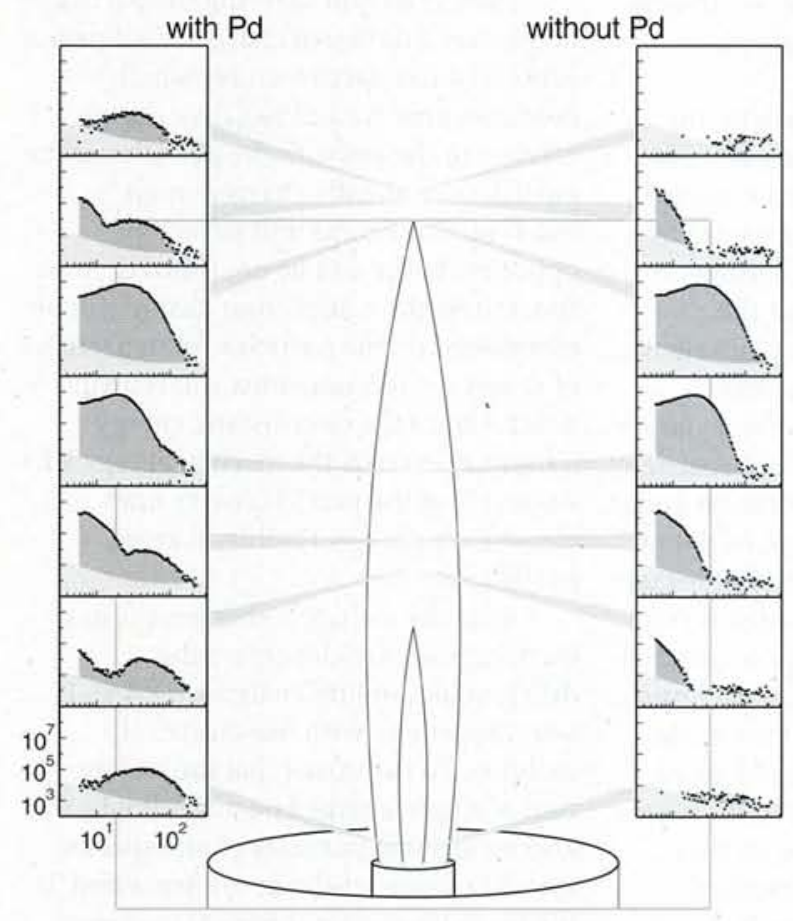

Fig. 6: Particle size distributions with samples taken at various heights in the flame. The left side represents the flame seeded with $\mathrm{Pd}$ particles, the right side the unseeded flame.

second DMA can be applied before admitting the particles to the aerosol electrometer.

First of all, one would like to test the extraction systems displayed in Fig. 1, particularly the question whether the particle size distribution measured in the extracted and diluted gas is the same as that in the flame. For this purpose, we added a test aerosol of known size distribution to the fuel gas and checked whether we could retrieve this test aerosol after extraction from the flame. The test aerosol was produced by heating a Pd wire in argon gas. The Pd vapour condenses in the cold Ar to form an aerosol with a size distribution of $10 \mathrm{~nm} \leq \mathrm{d} \leq 100 \mathrm{~nm}$. We then mixed the Ar with the methane flowing to the burner orifice. A particle filter can be inserted to remove the Pd particles without affecting the $\mathrm{CH}_{4} / \mathrm{Ar}$ mixture.

One can perform three different tests: (i) extract samples from the non-ignited $\mathrm{Ar} / \mathrm{CH}_{4}$ mixture, (ii) extract samples from the flame without Pd particles, and (iii) extract samples from the flame with the Pd particles added to the fuel gas. The summary of these tests is that the size distribution of the Pd particles remains the same with the exception of shrinkage of the large Pd particles in the flame. The large particles are agglomerates of a grapelike structure. The shrinkage occurs when these agglomerates are exposed to high temperatures. This has been observed before [12].
In Fig. 6 we show size distributions of particles in the flame at various heights above the burner orifice. On the left, $\mathrm{Pd}$ particles were added while on the right they were not. At $h=0$, we see the original spectrum of the Pd particles as generated by the hot Pd wire, while no particles are of course present in the unseeded flame. However, at $\mathrm{h}=30 \mathrm{~mm}$ one already observes the first very small particles in the flame at $d<20 \mathrm{~nm}$. As $\mathrm{h}$ increases to $40 \mathrm{~mm}$, the density and size of the particles formed in the flame increase dramatically, while the Pd particle presence is still discernible. At still larger heights, the density of flame-generated particles is so large that the Pd particles disappear in the background. Then, at $\mathrm{h}>70 \mathrm{~mm}$, first the large and later the small particles disappear and the Pd particle size distribution emerges from the background with only the largest particles affected [13].

The fact that the first particles appear at $\mathrm{h}=30 \mathrm{~mm}$ contradicts the standard model of soot formation. Figure 2 shows that the larger PAH are not even present at $\mathrm{h}=30 \mathrm{~mm}$. To explain the formation of the particles at $\mathrm{h}=30 \mathrm{~mm}$, a mechanism other than condensation of PAH must be active.

Furthermore, the internal structure of the particles must change at $\mathrm{h}=60 \mathrm{~mm}$. Figure 4 shows that fullerenes are obtained only from particles sampled at $\mathrm{h} \leq 60 \mathrm{~mm}$. It is indeed quite plausible that the part- icles change structure upon switching from the build-up to the burn-out mode.

\section{Surface properties of nascent soot} particles

The aim now is to investigate the surface properties and condensation of chemicals onto the particles nucleated in the flame. This is best done by measuring the photoemission of electrons from the particles in their natural gaseous environment. Our work differs from numerous other experiments in that we do not precipitate the particles onto a substrate prior to photoemission spectroscopy.

To introduce the spectroscopy of the photoelectric yield, in Fig. 7 we show results obtained with Pd particles of diameter $d=39 \mathrm{~nm}$. These particles were added to the fuel gas as described above. In the non-ignited gas flow, the photoelectric yield near photoelectric threshold does not change with the height above the burner orifice as expected. However, in the flame the Pd particles already start to reduce their yield from $\mathrm{h}=10 \mathrm{~mm}$ onwards. At about $30 \mathrm{~mm}$, the curve reaches its minimum of $1 / 5$ of the initial yield, but recovers to a high value on increasing h. From 40 to $60 \mathrm{~mm}$ height, the Pd particles are buried in the flamegenerated particles and cannot therefore be measured. When the Pd particles reappear at $70 \mathrm{~mm}$ height, they are again in the low yield phase [13].

Photoelectron yield spectroscopy can be interpreted on a phenomenological basis only. In any case, it is a very fine sensor of the surface properties, responding to adsorbates at coverages at and below the monolayer level. The surface of Pd has been studied in detail since it is one of the key catalysts of heterogeneous reactions. Based on the extensive experience with this model surface, we propose the following explanation for the observations plotted in Fig. 7. The initial decrease of the yield indicates the availability of carbon in the combustion. The formation of graphitic carbon at the surface of Pd is known to be able to induce a substantial reduction of the photoelectric yield, as observed. The subsequent increase of the yield at the onset of PAH formation indicates that PAH are adsorbed at the surface when the particles are in the photoemission tube. When the PAH have disappeared at the top of the flame, the yield is low because coverage of the Pd surface with graphitic carbon persists. This is consistent with the 


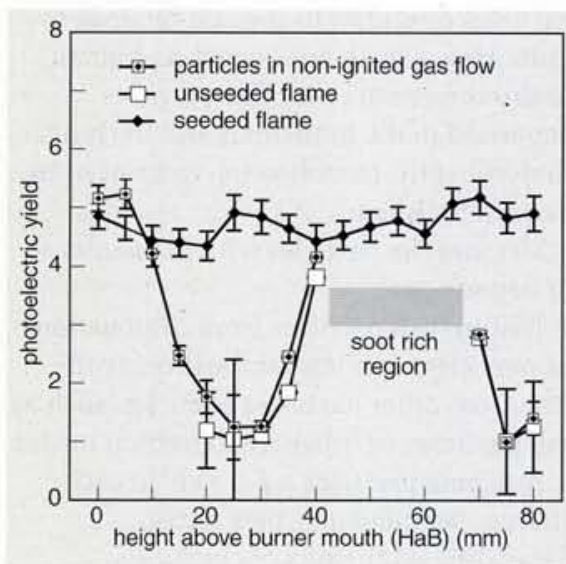

Fig. 7: Photoelectric yield in relative units from $\mathrm{Pd}$ particles with mobility diameter $39 \mathrm{~nm}$. The light source is a mercury discharge. The particles were extracted at the heights indicated from the non-ignited gas flow and from the flame. For comparison, results with the unseeded flame are also shown.

well-known fact that graphitic carbon, once deposited at the surface of Pd, is very hard to remove. In conclusion we see that the Pd surface conveys a picture of the chemistry in the flame, in particular it clearly marks the soot inception point at $\mathrm{h}=30 \mathrm{~mm}$, where carbon becomes available in large quantities.

We proceed now to the most interesting photoelectric yield of the particles generated in the flame. Figure 8 shows this yield for particles with $\mathrm{d}=35 \mathrm{~nm}$ as a function of the height above the burner orifice. The data labelled "bypass" are valid for particles as extracted and diluted with inert gas. The photoelectric yield of these particles is quite high from the moment they appear in concentrations sufficient for measurement. It hovers at a high level for extraction between 40 and $60 \mathrm{~mm}$ height, after which it plunges to reach $1 / 5$ of the maximum value at the end of the visible flame at $\mathrm{h}=70 \mathrm{~mm}$. The data labelled. "desorption" are valid for particles that have passed through a denuder prior to the measurement of the photoelectric yield. In the denuder, the gas carrying the particles is heated to $400{ }^{\circ} \mathrm{C}$, while the walls of the denuder contain active coal which absorbs any species that have been desorbed from the particles. We see in Fig. 8 that the photoelectric yield of the "naked" particles stripped of the adsorbates they acquired in the flame, or afterwards in the diluted gas stream, is generally lower by an order of magnitude. Moreover, it shows a different dependence on the height compared to the particles with adsorbates. The maximum yield occurs at $\mathrm{h}=60 \mathrm{~mm}$, and this maximum is sharp and reminiscent of the height profile maximum obtained with $\mathrm{C}_{60}$ as shown in Fig. 4 [14].

The interpretation is quite obvious from the many experimental observations we have made over the years in our laboratory: the yield of the adsorbate-covered particles is high due to the adsorption of PAH. Indeed, the yield curve follows the integrated height profile of the $\mathrm{PAH}$ [7]. However, in the case of the "naked" particles, the photoelectric yield curve is similar to the yield of fullerenes from the particles. Hence, the photoelectric yield of the "naked" particles demonstrates once more that the carbon clusters undergo a change in structure depending on the height above the burner orifice. The surface of highly oriented pyrolytic graphite (HOPG) has been extensively investigated by photoemission of electrons. It exhibits a very low photoelectric yield of electrons near the photoelectric threshold of $4.8 \mathrm{eV}$. The yield increases as the number of defects and dangling bonds at the surface increases [15]. This might be related to the carbon clusters: in the areas of rigorous growth there must be dangling bonds and defects at the surface, as a clean graphitic surface is inert and cannot bind any arriving species. But in the areas of the burnout, these active sites are destroyed first. Therefore, the photoelectric yield maximum separates the growth from the burn-out mode.

R. Schlögl and collaborators have been able to obtain TEM pictures from commercial soot and "fullerene" soot [16]. These pictures show the graphitic planes in the commercial soot but different, strandlike structures in the fullerene soot. The fullerene soot transforms into graphitic soot within hours. Hence we have to conclude that nascent soot is chemically different from old soot such as that collected on a filter.

Lastly, we discuss a detailed study of the desorption of PAH from gas suspended particles. The adsorption and desorption of combustion generated $\mathrm{PAH}$ on respiratory carrier particles is crucial for estimating the impact of combustion products on human health.

Aerosol photoemission makes it possible to observe directly the time dependence of the desorption of species from a surface. Ch. Hüglin has constructed a transport mechanism that can insert a small volume of gas containing particles into a particle-free stream of gas at higher temperature [17]. While the particles initially in a gas at low temperature are covered with the adsorbate at the instant of insertion into the hot gas, the adsorbates will start to evaporate as soon as they are in the hot gas environment. Photoemission of electrons induced by a sufficiently weak laser pulse, triggered a defined time after insertion of the particles into the hot gas, will detect the extent to which the adsorbates have desorbed because the photoelectric yield depends on the coverage of the surface.

Figure 9a shows results from a model experiment in which we produced graphite particles in a spark generator and guided the gas stream carrying these particles into a tube in which the partial pressure of perylene was kept high by evaporation from an oven. In this way, the graphite particles adsorb some perylene in the submonolayer range. Perylene is not chronically toxic, but it is an isomer of the carcinogen $\mathrm{B}(\mathrm{a}) \mathrm{P}$ and therefore can closely simulate the adsorption characteristics of this dangerous chemical. Figure ga shows that the desorption of

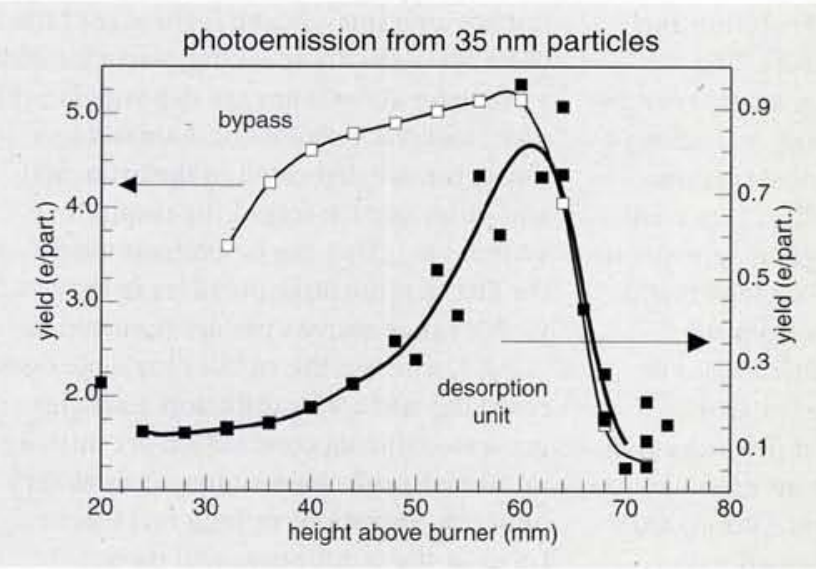

Fig. 8: Photoelectric yield of particles generated in the flame, extracted with system b) in Fig. 1, and with mobility diameter $35 \mathrm{~nm}$. The dilution with $\mathrm{N}_{2}$ was $1: 10$, the light source used for photoemission of electrons was a mercury discharge. The curve labelled "bypass" is for untreated particles, the curve labelled "desorption" after passing the gas carrying the particles through a denuder kept at $400^{\circ} \mathrm{C}$. 


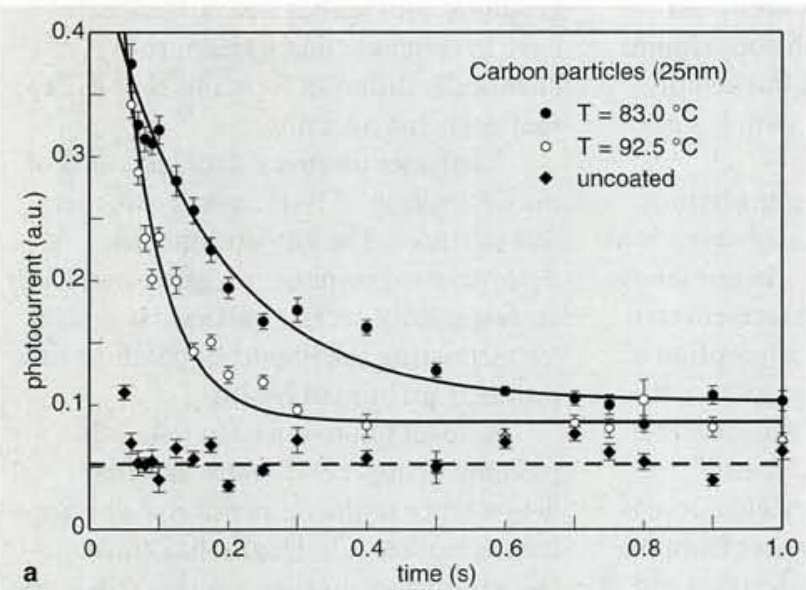

a

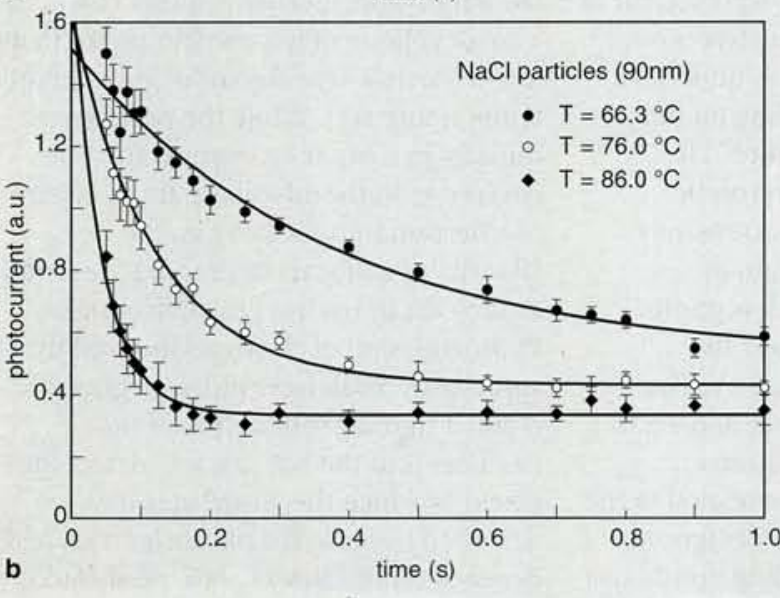

Fig. 9: Time dependence of the desorption of the 5 ring PAH perylene at various temperatures as observed with a pulsed laser beam of $6 \mathrm{eV}$ photons: a) for graphite particles of mobility diameter $d=25 \mathrm{~nm}$; $E_{d}=(88 \pm 13) \mathrm{kJ} / \mathrm{mole}$. b) for $\mathrm{NaCl}$ particles of $\mathrm{d}=98 \mathrm{~nm}, \mathrm{E}_{\mathrm{d}}=(123 \pm 28)$ $\mathrm{kJ} / \mathrm{mole}$. perylene depends exponentially on time, as expected. However, it is not possible to describe the observed desorption with a single adsorption site, rather one has to assume that at least two different adsorption sites exist. The heat of adsorption $\mathrm{E}_{\mathrm{d}}=(88 \pm 13) \mathrm{kJ} / \mathrm{mole}$ is in good agreement with other work if one takes into account that at least two adsorption sites exist [18 ]. Also important in the atmosphere are salt particles. In Fig. $9 \mathrm{~b}$ the desorption of perylene from $\mathrm{NaCl}$ particles is shown [17].These particles are produced by spraying an aqueous $\mathrm{NaCl}$ solution and removing the water in a dryer. The resulting $\mathrm{NaCl}$ particles are single crystals of a cubic shape. In this case, one adsorption site can describe the observations. The adsorption energy is $\mathrm{E}_{\mathrm{d}}=(123 \pm 28)$ $\mathrm{kJ} / \mathrm{mole}$, which is in very good agreement with previous work [19]. Perylene might gain energy by desorbtion from the combustion generated particles and readsorbtion on other particles. Our measurements suggest that the process of desorption and readsorption may take time of the order of $10 \mathrm{~min}$. Although the measurements were performed with perylene, one has to assume that $\mathrm{B}(\mathrm{a}) \mathrm{P}$ will behave in the same manner. With this mechanism, a particle which is harmless for human health taken by itself may become the carrier of a carcinogen. Figures $9 \mathrm{a}$ and $9 \mathrm{~b}$ also show clearly that with both carbon and $\mathrm{NaCl}$ particles $\mathrm{PAH}$ adsorbed on the surface are dominant in determining the photoelectric yield.

\section{Quantification of the health risk}

The key factor for evaluating the impact on human health is the size of the particles. Roughly speaking, particles with a diameter above $1 \mu \mathrm{m}$ are deposited in the nose, whereas particles of diameter less than $1 \mu \mathrm{m}$ are deposited in the bronchial and pulmonary tracts of the respiratory system [20]. This can be understood by the fact that the large particles in the visible range above $1 \mu \mathrm{m}$ are removed by impact, whereas the smaller invisible ones reach the surface by diffusion. Particles generated during combustion are initially in the size range below $1 \mu \mathrm{m}$. Only at very high concentrations or long residence times in the atmosphere will these particles coagulate to the size range above $1 \mu \mathrm{m}$. Hence the main impact on human health is from the very fine particles deposited in the bronchials and the lung. Consequently, the following tasks need to be accomplished:

1. Measure the particles with diameters at or below $1 \mu \mathrm{m}$.

2. Distinguish particles from combustion or particles carrying combustion products from other harmless particles, such as salt particles, or other condensation nuclei. 3. Attribute particles separately to each source - as there may be a factor depending on the mix of chemicals characteristic for each source.

4. Compare with the particles in cigarette smoke, since the danger from this source is best quantified so far.

5. Measure personal exposure rather than concentration of particles at a fixed location.

These requirements preclude many of the commonly-used techniques such as: counting all particles; measuring the mass of all particles; measuring the blackening of a filter; sampling methods with long sampling times as they cannot distinguish the sources; any method that is unable to detect the main killer, which is cigarette smoke.

We previously proposed that the measurement of the photoelectric charge density generated by irradiating a volume of air with the light from an $\mathrm{Hg}$ discharge or an excimer lamp, for instance, is eminently suited to determine human exposure to combustion products [21]. The two main reasons are that photoelectric charging occurs for particles smaller than $1 \mu \mathrm{m}$ only, hence the relevant size range is automatically selected, and that photoelectric charging is dominated by the $\mathrm{PAH}$ adsorbed on the particle surface. The fact that photoemission selects particles below $1 \mu \mathrm{m}$ in diameter is explained by the fact that recombination of the photoelectron with the positively charged particle is improbable when the mean free path of the electron exceeds the particle size. We have identified three different mechanisms which can explain the yield enhancement induced by adsorption of PAH [15]. The $\mathrm{PAH}$ are the most characteristic byproduct of the combustion of organic materials and contain the best investigated carcinogen $\mathrm{B}(\mathrm{a}) \mathrm{P}$ [2]. Therefore, adsorbed $\mathrm{PAH}$ are ideal tracers of combustion particles. There are also some practical reasons why one would like to use a sensor based on photoelectric charging: it can detect small concentrations of particle- 


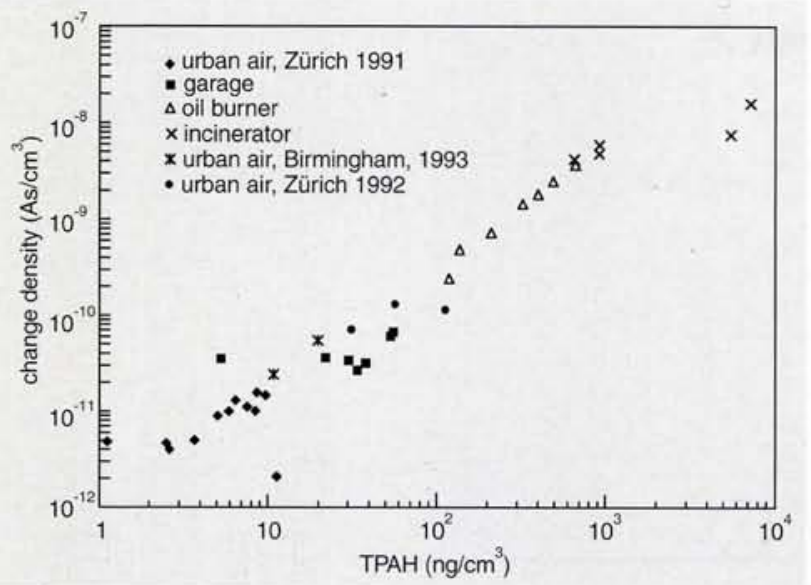

Fig. 10: Photoelectric charge density in Coulomb per $\mathrm{cm}^{3}$ as a function of total particle bound PAH concentration in $\mathrm{ng} / \mathrm{cm}^{3}$. See also Ref. [22]. bound $\mathrm{PAH}$, it is a continuous method, and it can also be constructed in a portable, battery operated form as we have demonstrated. The latter feature allows the individual exposure to be determined and the relation of this exposure to the actual state of health. In Fig. 10 we show the comparison of the gas-chromatographic chemical analysis of filter extracts with the photoelectric charge density. The remarkable result is that over a large range of concentrations, and with very different aerosols, a linear relationship is found between the total mass of PAH adsorbed on the particles and the photoelectric charge density. This demonstrates that photoemission of electrons is able to monitor the presence of particle-bound $\mathrm{PAH}$ in ambient air. The detection limit is about $1 \mathrm{ng} / \mathrm{m}^{3}$ of total mass of particle bound $\mathrm{PAH}$, for a time resolution of the order of $1 \mathrm{~s}$ [22].

As the impact on human health from smoking cigarettes is well known, instead of $\mathrm{ng} / \mathrm{m}^{3}$, we propose a unit that can instantly be interpreted: the daily cigarette exposure equivalent [DACEE]. To do this, we define a standard cigarette smoker. Naturally, any real smoker may be different from this standard smoker, yet we believe our definition comes close to reality for someone smoking contempo-

\section{Personal exposure of a physicist in various locations}

\begin{tabular}{|c|c|c|c|}
\hline $\begin{array}{l}\text { Live in countryside in Switzerland, } \\
\text { travel by train to office near motorway }\end{array}$ & 5.12 .95 & 0.6 & DACEE \\
\hline $\begin{array}{l}\text { Live in the city of Zürich, travel by public } \\
\text { transportation to office in countryside (ETH-Höngg) }\end{array}$ & 6.3 .96 & 1.2 & DACEE \\
\hline Vacations at lake Zürich near "Seestrasse" & Spring 96 & 2.0 & DACEE \\
\hline $\begin{array}{l}\text { Live in San Francisco, travel by car on } 280 \\
\text { to work at Stanford University }\end{array}$ & 2.9 .94 & 3.6 & DACEE \\
\hline $\begin{array}{l}\text { Live in Hotel in Tokyo, walk to office } \\
\text { at the University of Tokyo' }\end{array}$ & 16.1 .96 & 3.8 & DACEE \\
\hline Live in Campus at Tsinghua University, Bejing ${ }^{2}$ & 20.4 .96 & 2.7 & DACEE \\
\hline $\begin{array}{l}\text { Average daily exposure to combustion particles } \\
\text { of total mass } 10 \mu \mathrm{g} / \mathrm{m}^{3 *}\end{array}$ & & 5.5 & DACEE \\
\hline $1 \mathrm{~h}$ in street tunnel (Gubrist) near Zürich. & & 6.0 & DACEE \\
\hline \multicolumn{4}{|c|}{$\begin{array}{l}\text { *Note: Harvard six cities study shows that death rate due to cardiopulmonary disease rises by } 1 \% \text { a day } \\
\text { when there is an increase in the fine particle loading of } 10 \mu \mathrm{g} / \mathrm{m}^{3} \\
{ }^{1} \text { About } 30 \% \text { of this exposure is from cigarette smoke } \\
\text { }{ }^{3} \text { This is Saturday } 6 \text { a.m. to Sunday } 6 \text { a.m. Most of the exposure is at night when delivery trucks are allowed } \\
\text { run. }\end{array}$} \\
\hline
\end{tabular}

*Note: Harvard six cities study shows that death rate due to cardiopulmonary disease rises by $1 \%$ a day when there is an increase in the fine particle loading of $10 \mu \mathrm{g} / \mathrm{m}^{3}$

${ }^{1}$ About $30 \%$ of this exposure is from cigarette smoke

${ }^{2}$ This is Saturday 6 a.m. to Sunday 6 a.m. Most of the exposure is at night when delivery trucks are allowed to run.

\section{Acknowledgements}

We would like to acknowledge the help of Bingzhang Xue and Ernest Weingartner for the measurement of personal exposure. We are also grateful to H. Hepp, Ch. Hüglin, and M. Kasper for supplying results prior to publication.

\section{References}

[1.] D.W. Dockery et al., New Engl. J. Med. 329 (1993) 1753-1759

[2.] M.F. Denissenko, A. Pao, M.S. Tang, and G.P. Pfeifer, Science 274 (1996) 430-432

[3.] Physics Today May 1996, p. 63

[4.] H. Hepp, Diss. Nr. 11666, ETHZ 1996 [5.] M. Kasper, K. Siegmann, and K. Sattler, subm. to J. of Aerosol Sci. (1996)

[6.] T. Allain, S. Leach, and E. Sedlmayr, Astron. Astrophys. 305 (1996) 602-612 and 616-630, and Michel Brayer et al., Chem. Phys. Lett. 198 (1992) 128-134

[7.] K. Siegmann, H. Hepp, and K. Sattler, Combust. Sci. and Tech. 109 (1995) 165-181 [8.] M. Kasper, H. Hepp, and K. Siegmann, subm. to J. of Aerosol Sci. (1996)

[9.] K. Siegmann, H. Hepp, and K. Sattler, Surface Rev. and Lett. 3 (1996) 741

[10.] J.T. McKinnon and J.B. Howard, Combust. Sci. and Tech. 74 (1990) 175

[11.] K.-H. Homann, Bull. Soc. Chim. Belg. 99 (1990) 441-449

[12.] A. Schmidt-Ott, J. Aerosol Sci. 19 (1988) 553-563

[13.] M. Kasper, K. Siegmann, and K. Sattler, subm. to J. of Aerosol Sci. (1996)

[14.] H. Hepp and K. Siegmann, subm. to Combustion and Flame (1996)

[15.] T. Greber, T. Giessel, C. Pettenkofer, H.C. Siegmann, G. Ertl, Surf. Sci. Lett. 343 (1995) 1187-1191

[16.] Thilo Belz et al., Angew. Chem. Int. Ed. Engl. 33 (1994) 1866-1869

[17.] Ch. Hüglin, Diss. Nr. 11975, ETHZ 1996

[18.] D. Steiner and H.K. Burtscher, Environ. Sci. Technol. 28 (1994) 1254-1259

[19.] M.E. Loepfe, Diss. Nr. 9873, ETHZ 1992 [20.] W.C. Hinds, Sem. Resp. Med. 1 (1980) 197-210

[21.] H.C. Siegmann, Vierteljahresschrift der Naturforsch. Ges. in Zürich 135 (1990) 197-219 [22.]H. Burtscher and H.C. Siegmann, Combust. Sci. and Tech. 101 (1994) 327-332. 